\title{
THE TRANSMUTED GENERALIZED ODD GENERALIZED EXPONENTIAL-G FAMILY OF DISTRIBUTIONS: THEORY AND APPLICATIONS
}

\author{
Hesham Reyad $^{1 *}$, Soha Othman ${ }^{2}$, Muhammad Ahsan ul Haq ${ }^{3,4}$ \\ ${ }^{1}$ Department of Management Information Systems and Production \\ Management, Qassim University \\ ${ }^{2}$ Department of Applied Statistics, Cairo University \\ ${ }^{3}$ College of Statistical \& Actuarial Sciences, University of the Punjab \\ ${ }^{4}$ Quality Enhancement Cell, National College of Arts
}

\begin{abstract}
We propose a new generator of continuous distributions, so called the transmuted generalized odd generalized exponential-G family, which extends the generalized odd generalized exponential-G family introduced by Alizadeh et al. (2017). Some statistical properties of the new family such as; raw and incomplete moments, moment generating function, Lorenz and Bonferroni curves, probability weighted moments, Rényi entropy, stress strength model and order statistics are investigated. The parameters of the new family are estimated by using the method of maximum likelihood. Two real applications are presented to demonstrate the effectiveness of the suggested family.
\end{abstract}

Keywords: Odd Generalized Exponential-G family, Maximum Likelihood, Order Statistic, Stress Strength Model, Transmuted-G family.

\footnotetext{
* Corresponding author

Email:hesham_reyad@yahoo.com
} 


\section{Introduction}

In the last two decades, statisticians have introduced new classes of univariate distributions. These new families are formulated by adding new shape parameter(s) to the parent distribution to get more flexibility in fitting data in different fields such as medical sciences, economics, finance and environmental sciences. Some of the well-known generated families are the following: Marshall-Olkin-G family by Marshall and Olkin (1997), exponentiated-G by Gupta et al. (1998), beta-G by Eugene et al. (2002), Kumaraswamy-G by Cordeiro and de Castro (2011), McDonald-G by Alexander et al. (2012), logistic-G by Torabi and Montazari (2014), Lomax-G by Cordeiro et al. (2014), Kumaraswamy Marshall-Olkin-G by Alizadeh et al. (2015), odd-Burr generalized-G by Alizadeh et al. (2016), beta weibull-G by Yousof et al. (2017), Type I general exponential class of distributions by Hamedani et al. (2017), beta transmuted-H family by Afify et al. (2017), Topp-Leone odd log-logistic family by de Brito et al.(2017), T-Pareto-G by Hamed et al. (2018), among others.

Let $g(x, \phi)$ and $G(x ; \phi)$ denote the probability density function (pdf) and cumulative distribution function (cdf) of a baseline model with parameter vector $\phi$. Shaw and Buckley (2007) introduced the transmuted-G (T-G) family of distributions with cdf and pdf given by

$$
F(x ; \phi)=H(x ; \phi)[1+\lambda-\lambda H(x ; \phi)], x \in R,
$$

and

$$
f(x ; \phi)=h(x ; \phi)[1+\lambda-2 \lambda H(x, \phi)], x \in R .
$$

respectively, where, $|\lambda| \leq 1$, is a shape parameter, $x>0$ and $\phi$ is the vector of parameters for the baseline cdf $G(x ; \phi)$. The T-G density is a mixture of the baseline density and the exponentiated-G (Exp-G) density with power parameter two. If $\lambda=0$, then the T-G density reduced to the baseline density. Moreover, Alizadeh et al. (2017) defined the generalized odd generalized exponential-G (GOGE-G) family of distributions with cdf and pdf given, respectively, by

$$
H(x, \phi)=\left(1-e^{\frac{-G(x ; \phi)^{\alpha}}{1-G(x ; \phi)^{\alpha}}}\right)^{\beta}, \alpha>0, \beta>0, x \in R
$$

and

$$
h(x ; \phi)=\frac{\alpha \beta g(x ; \phi) G(x ; \phi)^{\alpha-1}}{\left(1-G(x ; \phi)^{\alpha}\right)^{2}} e^{\frac{-G(x, \phi)^{\alpha}}{1-G(x ; \phi)^{\alpha}}}\left(1-e^{\frac{-G(x ; \phi)^{\alpha}}{1-G(x ; \phi)^{\alpha}}}\right)^{\beta-1}, x \in R
$$


In this paper, we introduce a new class of continuous distributions called the transmuted generalized odd generalized exponential-G (TGOGE-G for short) family by using the GOGE $-\mathrm{G}$ as baseline distribution in the T-G generator and study some of its statistical properties The cdf and pdf of the TGOGE-G family are given, respectively, by

$$
F(x ; \alpha, \beta, \lambda)=(1+\lambda)\left(1-e^{\frac{-G(x ; \phi)^{\alpha}}{1-G(x ; \phi)^{\alpha}}}\right)^{\beta}-\lambda\left(1-e^{\frac{-G(x ; \phi)^{\alpha}}{1-G(x ; \phi)^{\alpha}}}\right)^{2 \beta}
$$

and

$$
\begin{aligned}
f(x ; \alpha, \beta, \lambda) & =\frac{\alpha \beta g(x ; \phi) G(x ; \phi)^{\alpha-1}}{\left(1-G(x ; \phi)^{\alpha}\right)^{2}} e^{\frac{-G(x ; \phi)^{\alpha}}{1-G(x ; \phi)^{\alpha}}}\left(1-e^{\frac{-G(x ; \phi)^{\alpha}}{1-G(x ; \phi)^{\alpha}}}\right)^{\beta-1} \\
& \times\left\{1+\lambda-2 \lambda\left(1-e^{\frac{-G(x ; \phi)^{\alpha}}{1-G(x ; \phi)^{\alpha}}}\right)^{\beta}\right\}, x \in R,
\end{aligned}
$$

Henceforth, a random variable with density (6) is denoted by $\operatorname{XDTGOGE}(\alpha, \beta, \lambda, \phi)$. The hazard function $\tau(x)$ for the TGOGE-G family is given by

$$
\tau(x)=\frac{\frac{\alpha \beta g(x ; \phi) G(x ; \phi)^{\alpha-1}}{\left(1-G(x ; \phi)^{\alpha}\right)^{2}} e^{\frac{-G(x ; \phi)^{\alpha}}{1-G(x ; \phi)^{\alpha}}}\left(1-e^{\frac{-G(x ; \phi)^{\alpha}}{1-G(x ; \phi)^{\alpha}}}\right)^{\beta-1}\left\{1+\lambda-2 \lambda\left\{e^{\frac{-G(x ; \phi)^{\alpha}}{1-G(x ; \phi)^{\alpha}}}\right\}^{\beta}\right\}}{1-(1+\lambda)\left(1-e^{\frac{-G(x ; \phi)^{\alpha}}{1-G(x ; \phi)^{\alpha}}}\right)^{\beta}+\lambda\left(1-e^{\frac{-G(x ; \phi)^{\alpha}}{1-G(x ; \phi)^{\alpha}}}\right)^{2 \beta}} .
$$

The TGOGE-G has the following sub-families:

*If $\lambda=0$, then the TGOGE-G class reduces to the GOGE-G family.

*If $\lambda=0, \alpha=1$, then the TGOGE-G class becomes the OGE-G family introduced by Tahir et al. (2015).

*If $\lambda=0, \alpha=\beta=1$, then we have the OE-G family given by Bourgulnon et al. (2014)

*If $\alpha=1$, then we have the TOGE -G family (new)

*If $\alpha=\beta=1$, then we get the TOE-G (new). 
The primary inspiration of proposed family is to derive a new extension of the GOGE-G family by inducting one extra additional parameter with an aim of (1) acquire greater adaptability as for skewness, kurtosis, tail weight, and length, (2) Covering some important probability models and (3) Providing significant improvement in data modelling.

The rest of this paper is outlined as follows:. In Section 2, usefull expansion of TGOGE -G family is discussed. In Section 3, some special models corresponding to TGOGE-G are introduced. In Section 4, some statistical properties of the TGOGE -G are discussed. In Section 5, the maximum likelihood estimates are obtained for model parameters. In Section 6, two applications for TGOGE-G are presented. Some concluding remarks are given in the last Section.

\section{Useful expansions}

In this section, we introduce a useful expansion for the TGOGE-G pdf and cdf. The pdf given in (6) can be written as

$$
\begin{aligned}
f(x) & =\frac{\alpha \beta(1+\lambda) g(x ; \phi) G(x ; \phi)^{\alpha-1}}{\left(1-G(x ; \phi)^{\alpha}\right)^{2}} e^{\frac{-G(x ; \phi)^{\alpha}}{1-G(x ; \phi)^{\alpha}}}\left(1-e^{\frac{-G(x ; \phi)^{\alpha}}{1-G(x ; \phi)^{\alpha}}}\right)^{\beta-1} \\
& -\frac{2 \alpha \beta \lambda g(x ; \phi) G(x ; \phi)^{\alpha-1}}{\left(1-G(x ; \phi)^{\alpha}\right)^{2}} e^{\frac{-G(x ; \phi)^{\alpha}}{1-G(x ; \phi)^{\alpha}}}\left(1-e^{\frac{-G(x ; \phi)^{\alpha}}{1-G(x ; \phi)^{\alpha}}}\right)^{2 \beta-1}
\end{aligned}
$$

Using generalized binomial seiers in above equation, we can obtain

$$
f(x)=\sum_{j=0}^{\infty}(-1)^{j} \alpha \beta\left[(1+\lambda)\left(\begin{array}{c}
\beta-1 \\
j
\end{array}\right)-2 \lambda\left(\begin{array}{c}
2 \beta-1 \\
j
\end{array}\right)\right] \frac{g(x ; \phi) G(x ; \phi)^{\alpha-1}}{\left(1-G(x ; \phi)^{\alpha}\right)^{2}} e^{\frac{-(j+1) G(x ; \phi)^{\alpha}}{1-G(x ; \phi)^{\alpha}}}
$$

Appling Taylor expansion in last equation, we can get

$$
f(x)=\sum_{j, k=0}^{\infty} \frac{(-1)^{j+k} \alpha \beta(j+1)^{k}}{k !}\left[(1+\lambda)\left(\begin{array}{c}
\beta-1 \\
j
\end{array}\right)-2 \lambda\left(\begin{array}{c}
2 \beta-1 \\
j
\end{array}\right)\right] \frac{g(x ; \phi) G(x ; \phi)^{\alpha(k+1)-1}}{\left(1-G(x ; \phi)^{\alpha}\right)^{k+2}}
$$

Again, using generalized binomial series in last factor of the above equation, we have

$$
\begin{gathered}
f(x)=\sum_{j, k, \ell=0}^{\infty} \frac{(-1)^{j+k+\ell} \alpha \beta(j+1)^{k}}{k !}\left(\begin{array}{c}
-k-2 \\
\ell
\end{array}\right)\left[(1+\lambda)\left(\begin{array}{c}
\beta-1 \\
j
\end{array}\right)\right. \\
\left.-2 \lambda\left(\begin{array}{c}
2 \beta-1 \\
j
\end{array}\right)\right] g(x ; \phi) G(x ; \phi)^{\alpha(k+\ell+1)-1}
\end{gathered}
$$


or

$$
f(x)=\sum_{j=0}^{\infty} \sum_{k=0}^{\infty} \sum_{\ell=0}^{\infty} \pi_{j, k, \ell} h_{\alpha(k+\ell+1)}(x)
$$

where,

$$
\pi_{j, k, \ell}=\frac{(-1)^{j+k+\ell} \beta(j+1)^{k}}{(k+\ell+1) k !}\left(\begin{array}{c}
-k-2 \\
\ell
\end{array}\right)\left[(1+\lambda)\left(\begin{array}{c}
\beta-1 \\
j
\end{array}\right)-2 \lambda\left(\begin{array}{c}
2 \beta-1 \\
j
\end{array}\right)\right],
$$

and $h_{\alpha(k+\ell+1)}(x)=\alpha(k+\ell+1) g(x) G(x)^{\alpha(k+\ell+1)-1}$ is the exponentiated-G distribution with power parameter $\alpha(k+\ell+1)$.

By integrating from (8) with respect to $x$, we have

$$
F(x)=\sum_{j=0}^{\infty} \sum_{k=0}^{\infty} \sum_{\ell=0}^{\infty} \pi_{j, k, \ell} H_{\alpha(k+\ell+1)}(x)
$$

where, $H_{\alpha(k+\ell+1)}(x)=G(x)^{\alpha(k+\ell+1)}$.

\section{The TGOGE-G sub-models}

In this section, we introduce three special models of the TGOGE-G family.

\subsection{The TGOGE-Exponential (TGOGEE) Model}

The cdf and pdf of the exponential distribution are $G(x)=1-e^{-\theta x}$ and $g(x)=$ $\theta e^{-\theta x}, x>0, \theta>0$ respectively. Then, the cdf and pdf of TGOGE-Exponential (TGOGEE) are, respectively, given by

$$
\begin{aligned}
& f(x ; \alpha, \beta, \lambda, \theta)=\frac{\alpha \beta \theta e^{-\theta x}\left(1-e^{-\theta x}\right)^{\alpha-1}}{\left\{1-\left(1-e^{-\theta x}\right)^{\alpha}\right\}^{2}} e^{\frac{-\left(1-e^{-\theta x}\right)^{\alpha}}{\left(1-e^{-\theta x}\right)^{\alpha}}} \\
& \left(1-e^{\frac{-\left(1-e^{-\theta x}\right)^{\alpha}}{\left(1-e^{-\theta x}\right)^{\alpha}}}\right)^{\beta-1}\left\{1+\lambda-2 \lambda\left(1-e^{\frac{-\left(1-e^{-\theta x}\right)^{\alpha}}{\left(1-e^{-\theta x}\right)^{\alpha}}}\right)^{\beta}\right\},
\end{aligned}
$$

and

$$
F(x ; \alpha, \beta, \lambda, \theta)=\left(1-e^{\frac{-\left(1-e^{-\theta x}\right)^{\alpha}}{1-\left(1-e^{-\theta x}\right)^{\alpha}}}\right)^{\beta}\left\{1+\lambda-\lambda\left(1-e^{\frac{-\left(1-e^{-\theta x}\right)^{\alpha}}{1-\left(1-e^{-\theta x}\right)^{\alpha}}}\right)^{\beta}\right\}
$$


The plots of the density and hazard functions are given in Figure 1.
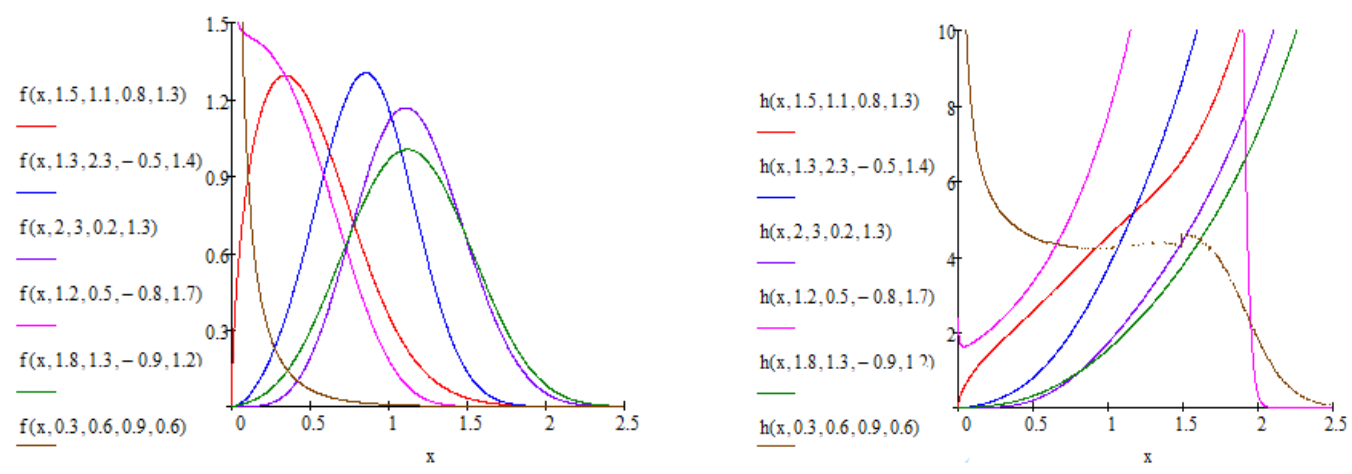

Figure 1: Plots of the TGOGEE pdf and hrf for selected values of parameters

\subsection{The TGOGE-Lomax (TGOGELx) model}

Consider the cdf and pdf of the Lomax distribution $G(x)=1-(1+b x)^{-a}$ and $g(x)=$ $a b(1+b x)^{-(a+1)}, x>0, a, b>0$ respectively. Then, the cdf and pdf of TGOGE-Lomax (TGOGELx) are, respectively, given by

$$
\begin{aligned}
f(x ; \alpha, \beta, \lambda, a, b) & =\frac{\alpha \beta a b(1+b x)^{-(a+1)}\left(1-(1+b x)^{-(a+1)}\right)^{\alpha-1}}{\left\{1-\left(1-(1+b x)^{-(a+1)}\right)^{\alpha}\right\}^{2}} e^{\frac{-\left(1-(1+b x)^{-a}\right)^{\alpha}}{1-\left(1-(1+b x)^{-a}\right)^{\alpha}}} \\
& \times\left(1-e^{\frac{-\left(1-(1+b x)^{-a}\right)^{\alpha}}{1-\left(1-(1+b x)^{-a}\right)^{\alpha}}}\right)^{\beta-1}\left\{1+\lambda-2 \lambda\left(1-e^{\frac{-\left(1-(1+b x)^{-a}\right)^{\alpha}}{1-\left(1-(1+b x)^{-a}\right)^{\alpha}}}\right)^{\beta}\right\}
\end{aligned}
$$

and

$$
F(x ; \alpha, \beta, \lambda, a, b)=\left(1-e^{\frac{-\left(1-(1+b x)^{-a}\right)^{\alpha}}{1-\left(1-(1+b x)^{-a}\right)^{\alpha}}}\right)^{\beta}\left\{1+\lambda-2 \lambda\left(1-e^{\frac{-\left(1-(1+b x)^{-a}\right)^{\alpha}}{1-\left(1-(1+b x)^{-a}\right)^{\alpha}}}\right)^{\beta}\right\}
$$

The plots of the density and hazard functions are given in Figure 2.
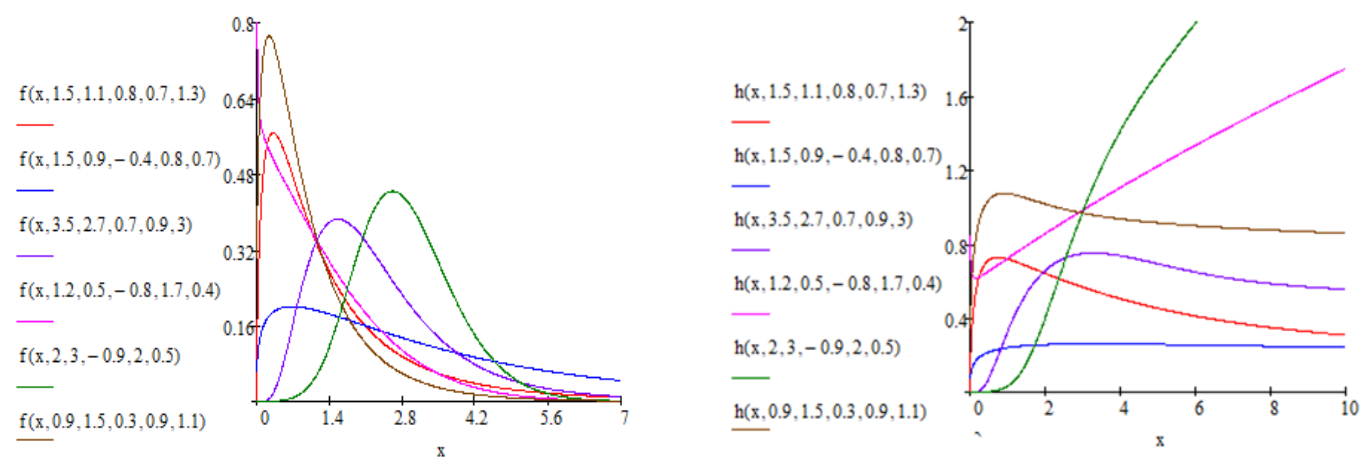
Figure 2: Plots of the TGOGELx pdf and hrf for selected values of parameters

\subsection{The TGOGE-Kumaraswamy (TGOGEKw) model}

The cdf and pdf of the Kumaraswamy distribution are $G(x)=1-\left(1-x^{b}\right)^{a}$ and $g(x)=a b x^{b-1} 1-\left(1-x^{b}\right)^{a}-1,0<x<1 \quad a, b>0$, respectively. Then, the cdf and pdf of TGOGE-Kumaraswamy (TGOGEKw) are, respectively, given by

$$
\begin{aligned}
f(x ; \alpha, \beta, \lambda, a, b) & =\frac{\alpha \beta a b x^{b-1}\left(1-x^{b}\right)^{a-1}\left(1-\left(1-x^{b}\right)^{a}\right)^{\alpha-1}}{\left\{1-\left(1-\left(1-x^{b}\right)^{a}\right)^{\alpha}\right\}^{2}} e^{\frac{-\left(1-\left(1-x^{b}\right)^{a}\right)^{\alpha}}{\left.1-\left(1-x^{b}\right)^{a}\right)^{\alpha}}} \\
& \times\left(1-e^{\frac{-\left(1-\left(1-x^{b}\right)^{a}\right)^{\alpha}}{1-\left(1-\left(1-x^{b}\right)^{a}\right)^{\alpha}}}\right)^{\beta-1}\left\{1+\lambda-2 \lambda\left(1-e^{\frac{-\left(1-\left(1-x^{b}\right)^{a}\right)^{\alpha}}{1-\left(1-\left(1-x^{b}\right)^{a}\right)^{\alpha}}}\right)^{\beta}\right\}
\end{aligned}
$$

and

$$
F(x ; \alpha, \beta, \lambda, a, b)=\left(1-e^{\frac{-\left(1-\left(1-x^{b}\right)^{a}\right)^{\alpha}}{1-\left(1-\left(1-x^{b}\right)^{a}\right)^{\alpha}}}\right)^{\beta}\left\{1+\lambda-2 \lambda\left(1-e^{\frac{-\left(1-\left(1-x^{b}\right)^{a}\right)^{\alpha}}{1-\left(1-\left(1-x^{b}\right)^{a}\right)^{\alpha}}}\right)^{\beta}\right\}
$$

The plots of the density and hazard function are given in Figure 3.
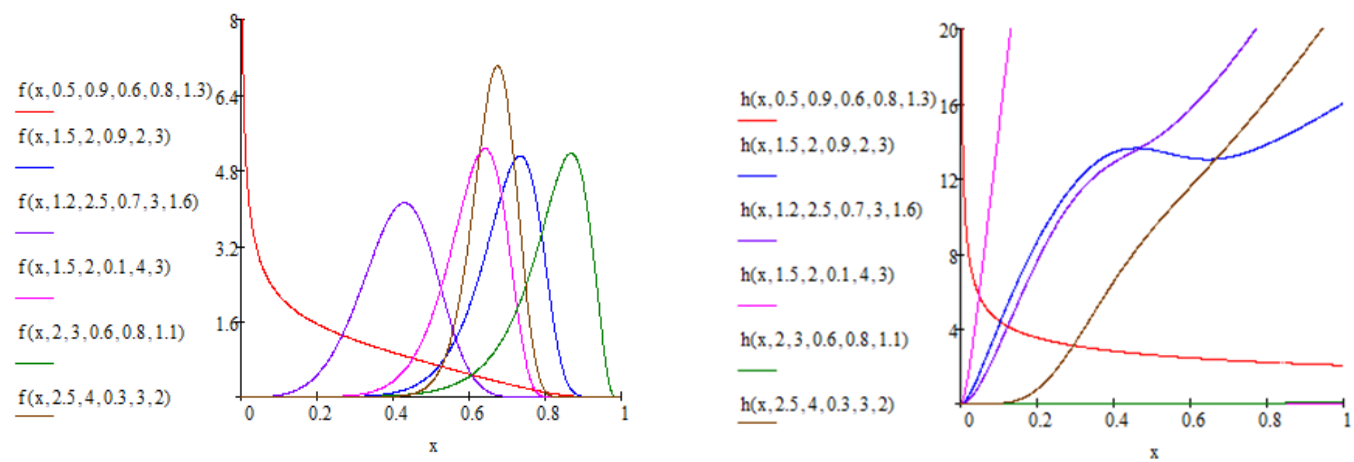

Figure 3: Plots of the TGOGEKx pdf and hrf for selected values of parameters 


\section{Statistical properties}

In this section, we investigate some statistical properties of the TGOGE-G family such as:quantile function, raw and incomplete moments, moment generating function, Lorenz and Bonferroni curves, Rényi entropy, stress strengt Statistical Properties h model and order statistics

\subsection{Quantile function}

The quantile function of the TGOGE-G family say $Q(u)=F^{-1}(u)$ for $u \in(0,1), \lambda \neq$ 0 and $\alpha \neq 0$ is the solution of the non-linear equation

$$
Q(u)=G^{-1}\left\{\frac{\log \left[1-\left(1+\lambda-\sqrt{(1+\lambda)^{2}-4 \lambda u}\right)^{1 / \beta}\right]}{\log \left[1-\left(1+\lambda-\sqrt{(1+\lambda)^{2}-4 \lambda u}\right)^{1 / \beta}\right]-1}\right\}^{1 / \alpha}
$$

\subsection{Probability weighted moments}

The PWM method can be used for estimating the model parameters of that distributions whose inverse form cannot be expressed in explicit form. The $(r+s)$ th PWM of $X$ has the TGOGE-G distribution, say, $M_{r, s}$ is given by

$$
M_{r, s}=E\left(X^{r} F(x)^{s}\right)=\int_{-\infty}^{\infty} X^{r} F(x)^{s} f(x) d x
$$

From (5) and (6), we can get

$$
f(x) F(x)^{s}=\sum_{j=0}^{\infty} \sum_{i=0}^{\infty} \sum_{w=0}^{\infty} \sum_{\ell=0}^{\infty} \eta_{j, i, w, \ell} g(x) G(x)^{\alpha(w+\ell+1)}
$$

where,

$$
\begin{gathered}
\eta_{j, k, h, \ell}=(-1)^{j+i+w+\ell}(w !)^{-1} \alpha \beta \lambda^{j}(1+\lambda)^{s-j}(i+1)^{w}\left(\begin{array}{c}
s \\
j
\end{array}\right)\left(\begin{array}{c}
-w-2 \\
\ell
\end{array}\right)[(1 \\
\left.+\lambda)\left(\begin{array}{c}
\beta(s-j+1)-1 \\
i
\end{array}\right)-2 \lambda\left(\begin{array}{c}
\beta(s-j+2) \\
i
\end{array}\right)\right]
\end{gathered}
$$


Therefore, we have

$$
M_{r, s}=\sum_{j=0}^{\infty} \sum_{i=0}^{\infty} \sum_{w=0}^{\infty} \sum_{\ell=0}^{\infty} \eta_{j, i, w, \ell} \psi_{r, \alpha(w+\ell+1)}
$$

where, $\psi_{r, \alpha(w+\ell+1)}=\int_{-\infty}^{\infty} x^{r} g(x) G(x)^{\alpha(w+\ell+1)} d x$ is the probability weighted moment of the parent distribution.

\subsection{Raw, incomplete moments and moment generating functions}

Consider $X$ is a random variable with TGOGE-G distribution, then the raw moments, say $\mu_{r}^{\prime}$, is given by

$$
\mu_{r}^{\prime}=E\left(X^{r}\right)=\int_{-\infty}^{\infty} x^{r} f(x) d x=\sum_{j=0}^{\infty} \sum_{k=0}^{\infty} \sum_{\ell=0}^{\infty} \pi_{j, k, \ell}^{*} \psi_{r, \alpha(k+\ell+1)-1}
$$

where, $\pi_{j, k, \ell}^{*}=\alpha(k+\ell+1) \pi_{j, k, \ell}$.

The nth central moment of the TGOGE-G distribution, say $\mu_{n}$ can be obtained from

$$
\mu_{n}=\sum_{r=0}^{n}\left(\begin{array}{l}
n \\
r
\end{array}\right)\left(-\mu_{1}^{\prime}\right)^{n-r} E\left(x^{r}\right)=\sum_{r=0}^{n} \sum_{j=0}^{\infty} \sum_{k=0}^{\infty} \sum_{\ell=0}^{\infty}\left(\begin{array}{l}
n \\
r
\end{array}\right)\left(-\mu_{1}^{\prime}\right)^{n-r} \pi_{j, k, \ell}^{*} \psi_{r, \alpha(k+\ell+1)-1}
$$

The $r t h$ incomplete moment of the TGOGE-G distribution, denoted by $\varphi_{w}(t)$, is

$$
\varphi_{w}(t)=\int_{-\infty}^{t} x^{w} f(x) d x=\sum_{j=0}^{\infty} \sum_{k=0}^{\infty} \sum_{\ell=0}^{\infty} \pi_{j, k, \ell}^{*} K_{w, \alpha(k+\ell+1)-1}
$$

where, $K_{w, \alpha(k+\ell+1)-1}=\int_{-\infty}^{t} x^{w} g(x) G(x)^{\alpha(k+\ell+1)-1} d x$.

The moment generating function, say $M_{x}(t)$, of the TGOGE-G distribution can be obtained to be

$$
M_{x}(t)=E\left(e^{t x}\right)=\sum_{j=0}^{\infty} \sum_{k=0}^{\infty} \sum_{\ell=0}^{\infty} \frac{t^{r}}{r !} \pi_{j, k, \ell}^{*} \psi_{r, \alpha(k+\ell+1)-1}
$$




\subsection{Order statistics}

Order statistics play an important role in probability and statistics. Let $X_{1, n} \leq$ $X_{2: n}, \ldots, X_{n: n}$ be the ordered sample from a population of size $n$ following the TGOGE-G family. The pdf of $X_{k: n}$, the $k t h$ order statistic is given by

$$
f_{X_{k: n}}(x)=\frac{1}{\beta(k, n-k+1)} \sum_{w=0}^{n-k}(-1)^{w}\left(\begin{array}{c}
n-k \\
w
\end{array}\right) f(x) F(x)^{k+w-1} .
$$

where, $\beta(.,$.$) is the beta function. Using (5) and (6) in the above equation and after some$ manipulation, we can obtain

$$
f_{X_{k: n}}(x)=\sum_{w=0}^{n-k} \sum_{j=0}^{\infty} \sum_{s=0}^{\infty} \sum_{h=0}^{\infty} \sum_{\ell=0}^{\infty} \Omega_{w, j, s, h, \ell} h_{\alpha(h+\ell+1)}(x)
$$

where,

$$
\begin{aligned}
\Omega_{w, j, s, h, \ell} & =\frac{(-1)^{j+s+h+\ell+w} \beta \lambda^{j}(1+\lambda)^{k+w-j}(1+s)^{h}}{(h+\ell+1) h ! \beta(k, n-k+1)}\left(\begin{array}{c}
n-k \\
w
\end{array}\right)\left(\begin{array}{c}
k+w-1 \\
j
\end{array}\right)\left(\begin{array}{c}
-h-2 \\
\ell
\end{array}\right) \\
& \times\left[(1-\lambda)\left(\begin{array}{l}
i \\
h
\end{array}\right)\left(\begin{array}{c}
\beta(k+w+j)-1 \\
s
\end{array}\right)-2 \lambda\left(\begin{array}{c}
\beta(k+w+j)-1 \\
s
\end{array}\right)\right],
\end{aligned}
$$

Moreover, the rth moment of $k t h$ order statistic for TGOGE-G family is given by

$$
E\left(x_{k: n}^{r}\right)=\sum_{w=0}^{n-k} \sum_{j=0}^{\infty} \sum_{s=0}^{\infty} \sum_{h=0}^{\infty} \sum_{\ell=0}^{\infty} \Omega_{w, j, s, h, \ell}^{*} \psi_{r, \alpha(h+\ell+1)-1}
$$

where, $\Omega_{w, j, s, h, \ell}^{*}=\alpha(h+\ell+1) \Omega_{w, j, s, h, \ell}$.

\subsection{Lorenz and bonferroni curves}

The Lorenz and Bonferroni curves have been used in different fields such as reliability, economics, demography, insurance and medicine. The Lorenz $L_{F}(x)$ and Bonferroni $B(F(x))$ curves are defined respectively as follows:

$$
L_{F}(x)=\frac{1}{E(x)} \int_{0}^{x} t f(t) d t, \quad B(F(x))=\frac{1}{F(x) E(x)} \int_{0}^{x} t f(t) d t=\frac{L_{F}(x)}{F(x)} .
$$


Therefore, these quantities for the TGOGE-G distribution are given below

$$
L_{F}(x)=\frac{\sum_{j=0}^{\infty} \sum_{k=0}^{\infty} \sum_{\ell=0}^{\infty} \pi_{j, k, \ell}^{*} K_{1, \alpha(k+\ell+1)-1}}{\sum_{j=0}^{\infty} \sum_{k=0}^{\infty} \sum_{\ell=0}^{\infty} \pi_{j, k, \ell}^{*} \Psi_{1, \alpha(k+\ell+1)-1}}
$$

and

$$
B(F(x))=\frac{\sum_{j=0}^{\infty} \sum_{k=0}^{\infty} \sum_{\ell=0}^{\infty} \pi_{j, k, \ell}^{*} K_{1, \alpha(k+\ell+1)-1}}{F(x) \sum_{j=0}^{\infty} \sum_{k=0}^{\infty} \sum_{\ell=0}^{\infty} \pi_{j, k, \ell}^{*} \Psi_{1, \alpha(k+\ell+1)-1}}
$$

\subsection{Rényi entropy}

The idea of entropy has been used in different area such as statistics, queuing theory and reliability estimation. The Rényi entropy is defined as

$$
I_{R}(X)=(1-\gamma)^{-1} \log \int_{-\infty}^{\infty} f(x)^{\gamma} d x, \quad \gamma>0, \gamma \neq 0,
$$

From (6), we obtain

$$
f(x)^{\gamma}=\sum_{j=0}^{\infty} \sum_{r=0}^{\infty} \sum_{w=0}^{\infty} \sum_{\ell=0}^{\infty} T_{j, r, w, \ell} g(x)^{\gamma} G(x)^{\alpha(\gamma+w+\ell)-\gamma}
$$

where,

$$
T_{j, r, w, \ell}=(-1)^{j+r+w+\ell}(w !)^{-1}(\alpha \beta \lambda)^{\gamma}(1+\lambda)^{\gamma-j}(\gamma+r)^{w}\left(\begin{array}{l}
\gamma \\
j
\end{array}\right)\left(\begin{array}{c}
\beta(\gamma+j)-\gamma \\
r
\end{array}\right)\left(\begin{array}{c}
-2 \gamma-w \\
\ell
\end{array}\right) .
$$

Consequently, the Rényi entropy for the TGOGE-G family is given by

$$
I_{R}(X)=(1-\gamma)^{-1} \log \left(\sum_{j=0}^{\infty} \sum_{r=0}^{\infty} \sum_{w=0}^{\infty} \sum_{\ell=0}^{\infty} T_{j, r, w, \ell} \int_{-\infty}^{\infty} g(x)^{\gamma} G(x)^{\alpha(\gamma+w+\ell)-\gamma} d x\right) .
$$

\subsection{Stress strength model}

The stress strength model is a common criterion used in different applications in physics and engineering such as strength failure and system collapse. Let $X_{1}$ and $X_{2}$ be two independent random variables with $\operatorname{TGOGE}\left(\alpha_{1}, \beta_{1}, \lambda_{1}, \phi\right)$ and $\operatorname{TGOGE}\left(\alpha_{2}, \beta_{2}, \lambda_{2}, \phi\right)$ distributions. Then, the stress strength model is given by

$$
R=\mathrm{P}_{\mathrm{r}}\left(X_{2}<X_{1}\right)=\int_{0}^{\infty} f_{1}\left(\alpha_{1}, \beta_{1}, \lambda_{1} ; \phi\right) F_{2}\left(\alpha_{2}, \beta_{2}, \lambda_{2} ; \phi\right) d x
$$


Using (5) and (6), we get

$$
\begin{aligned}
& f_{1}\left(\alpha_{1}, \beta_{1}, \lambda_{1} ; \phi\right) F_{2}\left(\alpha_{2}, \beta_{2}, \lambda_{2} ; \phi\right) \\
& =\sum_{j=0}^{\infty} \sum_{i=0}^{\infty} \sum_{w=0}^{\infty} \sum_{h=0}^{\infty} \sum_{s=0}^{\infty} \sum_{\ell=0}^{\infty} \xi_{j, i, w, h, s, \ell} g(x) G(x)^{\alpha_{1}(w+s-1)+\alpha_{2}(h+\ell),}
\end{aligned}
$$

where,

$$
\begin{gathered}
\xi_{j, i, w, h, s, \ell}=(-1)^{j+i+w+h+s+\ell}(w ! h !)^{-1} \alpha_{1} \beta_{1}\left(\begin{array}{c}
-w-2 \\
s
\end{array}\right)\left(\begin{array}{c}
-h \\
\ell
\end{array}\right)\left[\left(1+\lambda_{1}\right)\left(\begin{array}{c}
\beta_{1}-1 \\
j
\end{array}\right)\right. \\
\left.-2 \lambda_{1}\left(\begin{array}{c}
2 \beta_{1}-1 \\
j
\end{array}\right)\right] \times\left[\left(1+\lambda_{2}\right)\left(\begin{array}{c}
\beta_{2} \\
i
\end{array}\right)-\lambda_{1}\left(\begin{array}{c}
2 \beta_{2} \\
i
\end{array}\right)\right]
\end{gathered}
$$

Therefore, the stress strength model is given below

$$
R=\sum_{j=0}^{\infty} \sum_{i=0}^{\infty} \sum_{w=0}^{\infty} \sum_{h=0}^{\infty} \sum_{s=0}^{\infty} \sum_{\ell=0}^{\infty} \xi_{j, i, w, h, s, \ell}^{*}
$$

where, $\xi_{j, i, w, h, s, \ell}^{*}=\left[\alpha_{1}(w+s-1)+\alpha_{2}(h+\ell)\right]^{-1} \xi_{j, i, w, h, s, \ell}$.

\section{Maximum likelihood estimation}

This section determine the maximum likelihood estimates (MLEs) of the model parameters of the TGOGE-G family. Let $x_{1}, x_{2}, \ldots, x_{n}$ be an independent random sample from TGOGE-G family with set of parameters $\Theta=(\alpha, \beta, \lambda, \phi)^{T}$ then the corresponding log-likelihood function is given by

$$
\begin{aligned}
\ell=n[\log (\alpha) & +\log (\beta)]+\sum_{i=1}^{n} \log \left(g\left(x_{i}, \phi\right)\right)+(\alpha-1) \sum_{i=1}^{n} \log \left(G\left(x_{i}, \phi\right)\right) \\
& -2 \sum_{i=1}^{n} \log \left(1-G(x ; \phi)^{\alpha}\right)-\sum_{i=1}^{n}\left(\frac{G(x ; \phi)^{\alpha}}{1-G(x ; \phi)^{\alpha}}\right)+(\beta-1) \sum_{i=1}^{n} \log \left(\varepsilon_{i}\right) \\
& +\sum_{i=1}^{n} \log \left(\sigma_{i}\right),
\end{aligned}
$$

where, $\varepsilon_{i}=1-e^{\frac{-G(x ; \phi)^{\alpha}}{1-G(x ; \phi)^{\alpha}}}$ and $\sigma_{i}=1+\lambda-2 \lambda \varepsilon_{i}^{\beta}$. 
The components of the score vector $\nabla \ell=\left(\frac{\partial \ell}{\partial \alpha}, \frac{\partial \ell}{\partial \lambda}, \frac{\partial \ell}{\partial \beta} \frac{\partial \ell}{\partial \phi_{r}}\right)$ are the following:

$$
\begin{aligned}
\frac{\partial \ell}{\partial \alpha} & =\frac{n}{\alpha}+\sum_{i=1}^{n} \log \left(G\left(x_{i}, \phi\right)\right)+2 \sum_{i=1}^{n}\left(\rho_{i}\right)-\sum_{i=1}^{n}\left(\varpi_{i}\right)+(\beta-1) \sum_{i=1}^{n}\left(\frac{\varpi\left(1-\varepsilon_{i}\right)}{\varepsilon_{i}}\right) \\
& -2 \lambda \beta \sum_{i=1}^{n}\left(\frac{\varpi\left(1-\varepsilon_{i}\right) \varepsilon_{i}^{\beta-1}}{\sigma_{i}}\right),
\end{aligned}
$$

where,

$$
\begin{gathered}
\rho_{i}=\frac{G(x ; \phi)^{\alpha} \log (G(x ; \phi))}{1-G(x ; \phi)^{\alpha}} \text { and } \varpi_{i}=\frac{\rho_{i}}{1-G(x ; \phi)^{\alpha}} \\
\frac{\partial \ell}{\partial \lambda}=\sum_{i=1}^{n} \log \left(\frac{1-2 \varepsilon_{i}^{\beta}}{\sigma_{i}}\right), \\
\frac{\partial \ell}{\partial \beta}=\frac{n}{\beta}+\sum_{i=1}^{n} \log \left(\varepsilon_{i}\right)-2 \lambda \sum_{i=1}^{n}\left(\frac{\varepsilon_{i}^{\beta} \log \left(\varepsilon_{i}\right)}{\sigma_{i}}\right)
\end{gathered}
$$

and (for $r=1, \ldots, q$ )

$$
\begin{aligned}
\frac{\partial \ell}{\partial \phi_{r}} & =\sum_{i=1}^{n}\left(\frac{g_{r}^{\prime}\left(x_{i}, \phi\right)}{g_{r}\left(x_{i}, \phi\right)}\right)+(\alpha-1) \sum_{i=1}^{n}\left(\frac{G_{r}^{\prime}\left(x_{i}, \phi\right)}{G_{r}\left(x_{i}, \phi\right)}\right)+2 \alpha \sum_{i=1}^{n}\left(v_{i}\right)-\alpha \sum_{i=1}^{n}\left(z_{i}\right) \\
& +\alpha(\beta-1) \sum_{i=1}^{n}\left(\frac{z_{i}\left(1-\varepsilon_{i}\right)}{\varepsilon_{i}}\right)-2 \alpha \lambda \beta \sum_{i=1}^{n}\left(\frac{z_{i}\left(1-\varepsilon_{i}\right) \varepsilon_{i}^{\beta-1}}{\sigma_{i}}\right),
\end{aligned}
$$

where,

$$
\begin{aligned}
g_{r}^{\prime}\left(x_{i}, \phi\right) & =\left(\partial_{-} r\left(x_{-} i, \phi\right)\right) /\left(\partial \phi_{-} r\right), G_{r}^{\prime}\left(x_{i}, \phi\right)=\left(\partial G_{-} r\left(x_{-} i, \phi\right)\right) /\left(\partial \phi_{-} r\right), \\
v_{i} & =\frac{G(x ; \phi)^{\alpha-1} G_{r}^{\prime}\left(x_{i}, \phi\right)}{1-G(x ; \phi)^{\alpha}} \text { and } z_{i}=\frac{v_{i}}{1-G(x ; \phi)^{\alpha}} .
\end{aligned}
$$

The MLEs, say $\hat{\Theta}=(\hat{\alpha}, \hat{\lambda}, \beta, \hat{\xi})$ of $\Theta=(\alpha, \lambda, \beta, \xi)^{T}$ can be obtained by solving the system of nonlinear equations (23) through (26). These equations cannot be solved analytically and it needed iterative techniques such as Newton-Raphson algorithm. 


\section{Applications}

In this section, we introduce two application to real data to show the usefulness of the TGOGE-G family. We focus on the TGOGE-E and TGOGE-Lx distributions introduced in Subsections 3.1 and 3.2 respectively. The first data set consists of 63 observations of the strengths of $1.5 \mathrm{~cm}$ glass fibers, originally obtained by workers at the UK National Physical Laboratory. The data are: $0.55,0.93,1.25,1.36,1.49,1.52,1.58,1.61,1.64,1.68,1.73,1.81$, $2,0.74,1.04,1.27,1.39,1.49,1.53,1.59,1.61,1.66,1.68,1.76,1.82,2.01,0.77,1.11,1.28$, $1.42,1.5,1.54,1.6,1.62,1.66,1.69,1.76,1.84,2.24,0.81,1.13,1.29,1.48,1.5,1.55,1.61$, $1.62,1.66,1.7,1.77,1.84,0.84,1.24,1.3,1.48,1.51,1.55,1.61,1.63,1.67,1.7,1.78,1.89$. These data have also been analyzed by Haq et al. (2017).

The second data set (gauge lengths of $10 \mathrm{~mm}$ ) from Kundu and Raqab (2009). This data set consists of, 63 observations: 1.901, 2.132, 2.203, 2.228, 2.257, 2.350, 2.361, 2.396, 2.397, $2.445,2.454,2.474,2.518,2.522,2.525,2.532,2.575,2.614,2.616,2.618,2.624,2.659$, $2.675,2.738,2.740,2.856,2.917,2.928,2.937,2.937,2.977,2.996,3.030,3.125,3.139$, $3.145,3.220,3.223,3.235,3.243,3.264,3.272$, 3.294, 3.332, 3.346, 3.377, 3.408, 3.435, $3.493,3.501,3.537,3.554,3.562,3.628,3.852$, 3.871, 3.886, 3.971, 4.024, 4.027, 4.225, 4.395, 5.020.

The MLEs are computed using Quasi-Newton Code for Bound Constrained Optimization (L-BFGS-B) and the log-likelihood function evaluated. The goodness-of-fit measures; AIC (Akaike information criterion), BIC (Bayesian information criterion), Anderson-Darling $\left(A^{*}\right)$ and Cramér-von Mises $\left(W^{*}\right)$, AIC (Akaike information criterion), and BIC (Bayesian information criterion) are computed. The better model corresponds to smaller AIC, BIC, $A^{*}$ and $W^{*}$ values.

For the first data set, we compare in the TGOGE-E model with those of the Kumaraswamy Weibull (KwW) (Cordeiro et al. 2010), Weibull Frèchet (WFr) (Afify et al. 2016), Kumaraswamy Frèchet (KwF) (Mead and Abd-Eltawab 2014) and Beta Exponential (BE) (Nadarajah and Kotz 2006) distributions. Moreover, for the second data set, we compare in the TGOGE-Lx model with those of the transmuted Weibull Lomax (TWL) (Afify et al. 2015), Weibull Lomax (WLx) (Tahir et al. 2015), modified Beta Weibull (MBW) (Khan, M. N 2015) and MacDonald Lomax (McLx) (Lemonte and Cordeiro, 2013) distributions. The MLEs and some statistics of the models for the first second data sets are presented in Tables (1), (2), (3) and (4) respectively. 
Table 1: Estimated values for the first data set

\begin{tabular}{ccccc}
\hline Model & \multicolumn{4}{c}{ Estimates } \\
\hline $\operatorname{TGOGE}-\mathrm{E}(\alpha, \beta, \lambda, \mathrm{a})$ & 8.98368 & 1.38743 & 0.62951 & 1.83925 \\
$\operatorname{KwW}(\alpha, \beta, \mathrm{a}, \mathrm{b})$ & 0.09843 & 3.71307 & 1.77383 & 3.72081 \\
$\operatorname{WFr}(\mathrm{a}, \mathrm{b}, \alpha, \beta)$ & 1.47620 & 16.8560 & 0.38650 & 0.24360 \\
$\operatorname{KwFr}(\alpha, \beta, \mathrm{a}, \mathrm{b})$ & 0.29362 & 0.40833 & 26.6296 & 582557. \\
$\operatorname{BE}(\gamma, \lambda, \mathrm{a})$ & 0.41622 & 17.7227 & 20.7865 & -- \\
\hline
\end{tabular}

Table 2: Goodness of fit measures for first data set

\begin{tabular}{cccccc}
\hline Model & $\ell$ & AIC & BIC & $A^{*}$ & $W^{*}$ \\
\hline $\operatorname{TGOGE}-\mathrm{E}(\alpha, \beta, \lambda, \mathrm{a})$ & -16.0170 & 39.000 & 47.573 & 1.3410 & 0.2326 \\
$\operatorname{KwW}(\alpha, \beta, \mathrm{a}, \mathrm{b})$ & -16.3926 & 40.7852 & 49.3577 & 1.59965 & 0.29207 \\
$\operatorname{WFr}(\mathrm{a}, \mathrm{b}, \alpha, \beta)$ & -15.8311 & 39.6622 & 48.2347 & 1.5006 & 0.2753 \\
$\operatorname{KwFr}(\alpha, \beta, \mathrm{a}, \mathrm{b})$ & -17.1956 & 42.3913 & 50.9638 & 1.7445 & 0.309542 \\
$\operatorname{BE}(\gamma, \lambda, \mathrm{a})$ & -24.1527 & 54.3055 & 60.7349 & 3.08716 & 0.565848 \\
\hline
\end{tabular}
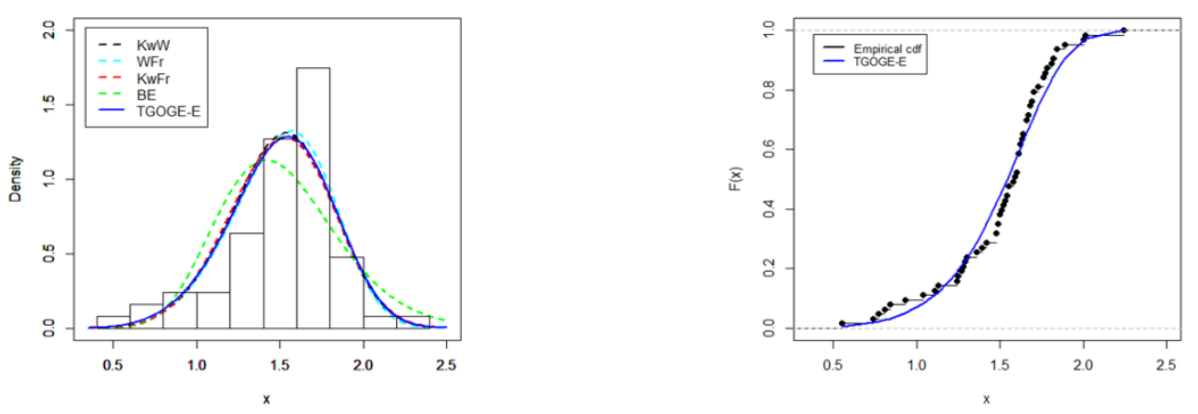

Figure 4: Estimated pdfs and cdfs plots of the TGOGEE distribution for data set 1 
DISTRIBUTIONS: THEORY AND APPLICATIONS

Table 3: Estimated values for the second data set

\begin{tabular}{cccccc}
\hline Model & \multicolumn{5}{c}{ Estimates } \\
\hline TGOGE-L $(\alpha, \beta, a, b, \delta)$ & 0.1397 & 637.2890 & 226.3480 & 0.0007 & 0.0775 \\
$\operatorname{TWL}(\alpha, \beta, \lambda, \mathrm{a}, \mathrm{b})$ & 7.6271 & 4.6868 & 0.7665 & 3.1524 & 21.1734 \\
$\operatorname{WL}(\alpha, \beta, \mathrm{a}, \mathrm{b})$ & 0.2471 & 0.3255 & 8.0030 & 10.1306 & --- \\
$\operatorname{MBW}(\alpha, \beta, \lambda, \mathrm{a}, \mathrm{b})$ & 1.8974 & 5.2354 & 17.2067 & 6.4617 & 0.0514 \\
$\operatorname{McL}(\alpha, \beta, \lambda, \mathrm{a}, \mathrm{b})$ & 45.9249 & 48.3024 & 353.1435 & 18.1192 & 195.4633 \\
\hline
\end{tabular}

Table 4: Goodness of fit measures for second data set

\begin{tabular}{cccccc}
\hline Model & $\ell$ & AIC & BIC & $A^{*}$ & $W^{*}$ \\
\hline TGOGE-L $(\alpha, \beta, \mathrm{a}, \mathrm{b}, \delta)$ & -56.2290 & 122.4580 & 133.1740 & 0.3256 & 0.0621 \\
$\operatorname{TWL}(\alpha, \beta, \lambda, \mathrm{a}, \mathrm{b})$ & -61.1920 & 132.3841 & 143.0998 & 0.8339 & 0.1205 \\
$\operatorname{WL}(\alpha, \beta, \mathrm{a}, \mathrm{b})$ & -60.8940 & 129.7870 & 138.3595 & 0.8122 & 0.1174 \\
$\operatorname{MBW}(\alpha, \beta, \lambda, \mathrm{a}, \mathrm{b})$ & -62.9580 & 135.9170 & 142.4895 & 1.0436 & 0.1517 \\
$\operatorname{McL}(\alpha, \beta, \lambda, \mathrm{a}, \mathrm{b})$ & -65.2980 & 140.5970 & 147.1695 & 0.8142 & 0.1081 \\
\hline
\end{tabular}
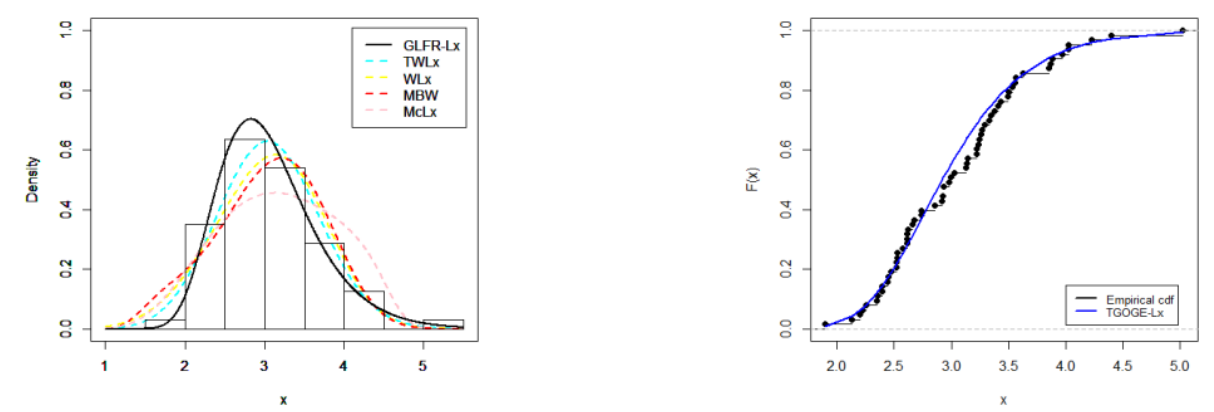

Figure 5: Estimated pdfs and cdfs plots of the TGOGELx distribution for data set 2

The values in Tables (2) and (4) showed that the TGOGEE and TGOGELx models have the lowest values for AIC, BIC, $\mathrm{A}^{*}$ and $\mathrm{W}^{*}$ among all fitted distributions in the first and second data sets respectively. So, they models could be chosen as the best fits. It is clear from Figures (4) and (5) that the new TGOGEE and TGOGELx distributions provides the best fits to the two data sets with compared with all competitive models. 


\section{Conclusions}

We propose a new class of distributions, called the transmuted generalized odd generalized exponential-G family. We investigate the statistical properties of the suggested family such as ordinary and incomplete moments, generating functions, Lorenz and Bonferroni curves, Rényi of entropy, stress strength model, and order statistics. The method of maximum likelihood is used to estimate the model parameters. Two real data sets are used to show that some models corresponding to the TGOGE-G family can give better fit than similar models generated by well-known families. 


\section{References}

[1] Afify, A. Z., Nofal, Z. M., Yousof, H. H., El Gebaly, Y. and Butt, N. S. (2015). The transmuted Weibull Lomax distribution: properties and application. Pak. J. Stat. Oper. Res. XI (1): 135-152.

[2] Afify, A. Z., Yousof, H. H., Cordeiro, G. M., Ortega, E. M. M. and Nofal, Z. M. (2016). The Weibull Frèchet distribution and its application. Journal of Applied Statistics, 43 (14): 2608-2626.

[3] Afify, A. Z., Yousof, H. H. and Nadarajah, S. (2017). The beta transmuted-H family of distributions:properties and applications. Statistics and its Interference, 10:505-520.

[4] Alexander, C., Cordeiro, G. M., Ortega, E. M. M., and Sarabia, J. M. (2012). Generalized beta-generated distributions. Comput. Stat. Data Anal, 56:1880-1897.

[5] Alizadeh, M., Cordeiro, G. M., Nascimento, A. D. C., Lima, M. D. S. and Ortega, E. M. M. (2016). Odd-Burr generalized family of distributions with some applications. Journal of Statistical Computation and Simulation, 83: 326-339.

[6] Alizadeh, M, Ghosh, I., Yousof, H. M., Rasekhi, M. and Hamedani, G. G. (2017). The generalized odd generalized exponential family of distributions: properties, characterizations and application. Journal of Data Science, 16: 443-466.

[7] Alizadeh, M, Tahir, M. H., Cordeiro, G. M., Mansoor, M., Zubair, M. and Hamedani, G. G. (2015). The Kumaraswamy Marshal-Olkin family of distributions. Journal of the Egyptian Mathematical Society, 23: 546-557.

[8] Bourguignon, M, Silva, R. B., Cordeiro, G. M. (2014). The Weibull-G family of probability distributions. J. Data Sci. 12: 53-68.

[9] Cordeiro, G. M., Ortega, E. M. M. and Nadarajah, S. (2010).The Kumaraswamy Weibull distribution with application to failure data. Journal of Franklin Institute. 247 (6): 1399-1429.

[10] Cordeiro, G. M. and de Castro, M. (2011). A new family of generalized distributions. Journal of Statistical Computation and Simulation, 81: 883-898.

[11] Cordeiro, G. M., Ortega, E. M. M., Popovic', B. V. and Pescim, R. R. (2014). The Lomax generator of distributions: properties, minification process and regression model. Appl. Math. Comput., 247: 465-486. 
[12] de Brito, E., Cordeiro, G. M., Yousof, H. M., Alizadeh, M. and Silva, G. O. (2017). Topp-Leone odd log-logistic family of distributions. Journal of Statistical Computation and Simulation,87(15): 3040-3058..

[13] Eugene, N., Lee, C., Famoye, F. (2002). Beta-normal distribution and its applications. Commun. Stat. Theory Methods, 31: 497-512.

[14] Gupta, R. C., Gupta, P. L. and Gupta, R. D. (1998). Modeling failure time data by Lehmann alternatives. Commun. Stat. Theory Methods, 27: 887-904.

[15] Hamed, D., Famoye, F. and Lee, C. (2018). On families of generalized Pareto distributions: properties and applications. Journal of Data Science, 16(2): 337-396.

[16] Hamedani, G. G., Yousof, H. M., Rasekhi, M., Alizadeh, M., Najibi, S. M. (2017). Type I general exponential class of distributions. International Journal of Applied \& Experimental Mathematics, 1-17.

[17] Haq, M. A., Yousof, H. M., \& Hashmi, S. (2017). A New Five-Parameter Frèchet Model for Extreme Values. Pakistan Journal of Statistics and Operation Research, 13(3), 617-632.

[18] Kundu, D., \& Raqab, M. Z. (2009). Estimation of $\mathrm{R}=\mathrm{P}(\mathrm{Y}<\mathrm{X})$ for three-parameter Weibull distribution. Statistics \& Probability Letters, 79(17), 1839-1846.

[19] Lemonte, A., Cordeiro, G. (2013). An extended lomax distribution. Statistics, 47: 800-816.

[20] Marshall, A. W. and Olkin, I. (1997). A new methods for adding a parameter to a family of distributions with application to the Exponential and Weibull families. Biometrika, 84: 641-652.

[21] Mead, M. E. and Abd-Eltawab, A. R. (2014). A note on Kumaraswamy Frechet distribution. Aust. J. Basic and Appl. Sci. 8: 294-300.

[22] Nadarajah, S., Kotz, S. (2006). The beta exponential distribution. Reliability Engineering\& System. 91 (6): 689-697.

[23] Shaw, W. T. and Buckley, I. R. C. (2007). The alchemy of probability distributions: beyond Gram-Charlier expansions and a skew-kurtotic-normal distribution from a rank transmutation map. Research report.

[24] Smith, R. L., \& Naylor, J. C. (1987). A comparison of maximum likelihood and Bayesian estimators for the three-parameter Weibull distribution. Applied Statistics, 358-369. 
[25] Tahir, M. H., Cordeiro, G. M., Mansoor, M. and Zubair, M. (2015). The Weibull-Lomax distribution:properties and applications. Hacettepe Journal of Mathematics and Statistics, 44(2):461-480

[26] ahir, M. M., Cordeiro, G. M., Alizadeh, M., Mansoor, M., Zubair, M. and Hamedani, G. G. (2015). The odd generalized exponrntial family of distributions with applications. Journal of Statistical Distributions and Applications.

[27] Torabi, H. and Montazari, N. H. (2014). The logistic-uniform distribution and its application, Commun. Stat.Simui. Comput, 43: 2551-2569.

[28] Yousof, H., Rasekhi, M., Afify, A. Z., Ghosh, I., Alizadeh, M. and Hamedani, G.G. (2017). The beta weibull-G family of distribution: Theory, characterizations and applications, Pakistan Journal of Statistics, 32(2): 95-116. 